\title{
The Hamilton-Jacobi analysis and Canonical Covariant description for three dimensional Palatini theory plus a Chern-Simons term
}

\author{
Alberto Escalante* and Aldair-Pantoja \\ Instituto de Física, Benemérita Universidad Autónoma de Puebla. \\ Apartado Postal J-48 72570, Puebla Pue., México,
}

(Dated: June 3, 2019)

By using the Hamilton-Jacobi [HJ] framework the three dimensional Palatini theory plus a ChernSimons term $[\mathrm{PCS}]$ is analyzed. We report the complete set of $H J$ Hamiltonians and a generalized $H J$ differential from which all symmetries of the theory are identified. Moreover, we show that in spite of PCS Lagrangian produces Einstein's equations, the generalized $H J$ brackets depend on a Barbero-Immirzi like parameter. In addition we complete our study by performing a canonical covariant analysis, and we construct a closed and gauge invariant two form that encodes the symplectic geometry of the covariant phase space.

PACS numbers: $98.80 .-\mathrm{k}, 98.80 . \mathrm{Cq}$

\section{INTRODUCTION}

Nowadays, the analysis of singular systems has been the cornerstone for studying all fundamental forces in nature. From the standard model, string theory, to canonical gravity and Loop Quantum Gravity there is a big effort for understanding the underlying symmetries of these systems 15]. In fact, these forces expose symmetries and it is mandatory to perform the study of these symmetries by using alternative frameworks beyond standard classical mechanics. In this respect, we can cite several approaches such as the Dirac-Bergman, Faddeev-Jackiw, Canonical Covariant and the Hamilton-Jacobi methods $[6$ 26]. The Dirac approach allows us to identify the constraints of singular systems, which are classified into first class and second class. The formed are generators of the gauge symmetry and the latter are used for constructing the Dirac brackets of the theory; with the constraints at hand the symmetries of the theory can be identified. Nonetheless, the classification between the constraints into first or second class is a difficult task, and alternative approaches can be required. In this respect, the Faddeev-Jackiw framework allows the construction of a symplectic tensor from which the symmetries of the theory can be identified. In the FJ framework it is not necessary to perform the classification of the constraints as in Dirac's method is done; for gauge systems in order to obtain the symplectic tensor it is necessary fixing the gauge, and this fact could complicate the analysis. On the other hand, the canonical covariant method is a symplectic approach based on the construction of a closed and gauge invariant symplectic two form. From the

*Electronic address: aescalan@ifuap.buap.mx

$\dagger$ Electronic address: jpantoja@ifuap.buap.mx 
symplectic two form we can perform a Hamiltonian analysis, however, in this approach we have not control on the constraints of the theory and relevant information of the symmetries can be missed; in addition, the constraints are useful for performing the counting of physical degrees of freedom, hence this step can not be carryout. Alternatively, the HJ approach developed by Güler is based on the construction of a fundamental differential defined on the phase space, and the fundamental blocks are the constraints of the theory called Hamiltonians. The HJ Hamiltonians can be involutives or noninvolutives and they are fundamental blocks for obtaining the characteristic equations, the gauge symmetries and the generalized HJ brackets. The construction of the fundamental differential is direct and the process for identifying the symmetries is in general more economical than in the other approaches; in this sense the HJ framework is an interesting alternative for analyzing gauge systems.

Along the ideas exposed above, the fundamental subject of this paper is to report the HJ and canonical covariant analysis for 3d gravity described in terms of Palatini's theory plus a ChernSimons term [PCS] coupled through an arbitrary Immirzi-like parameter called $\gamma$ [28]. It is wellknown that the addition of topological terms to physical actions does not modify the equations of motion, but there is a modification on the fundamental brackets; in this respect two theories sharing the same classical equations of motion do not are equivalents at all [29 31]. This fact is present in the four dimensional Holst action, described by Palatini's theory plus the addition of a topological term, the so-called Holst term [32]. In this respect, the equations of motion of Palatini's theory and Holst theory are the same, however, at Hamiltonian level the structures of the constraints of these theories are different; other examples concerned to this respect can be consulted in the following references 33 36]. Hence, we have a similar scenario for PCS theory; three dimensional Palatini and PCS theories share the same equations of motion, the fundamental brackets, however, are different. We use the Güler-HJ approach [23 27] because it is an elegant and economical framework for analyzing singular systems; in fact, we will extend those results reported in [36, 37]. On the other hand, we want to report alternative studies beyond Dirac's and Faddeev-Jackiw framework in order to have the best alternative for analyzing singular systems.

The paper is organized as follows. In the Section II we develop the HJ analysis for PCS theory. We construct a fundamental differential where the characteristics equations and all symmetries of the theory are found. We reproduce and extend the results reported in [36, 37]. In Section III the canonical covariant formalism is performed; we construct a closed and gauge invariant geometric structure from which a Hamiltonian description of the theory is developed, and we identify the symmetries of the theory, however, we comment the disadvantages of this formalism with respect the other ones reported in the literature. 


\section{HAMILTON-JACOBI ANALYSIS}

We start with the following action expressed as Palatini's 3d gravity theory plus a Chern Simons term [28]

$$
S[e, A]=2 \int_{M} e^{i} \wedge F_{i}+\frac{1}{\gamma} \int_{M}\left[2 A^{i} \wedge d A_{i}+\frac{2}{3} \varepsilon_{i j k} A^{i} \wedge A^{j} \wedge A^{k}\right]
$$

where $M$ is a three dimensional manifold without boundary, $F_{i}=d A_{i}+\frac{1}{2} \epsilon_{i j k} A^{j} \wedge A^{k}$ is the strength curvature of the 1 - form connexion $A^{i}$, the $e$ 's are the triad fields, $i, j, k . .=0,1,2$ are internal $S U(2)$ indices, and $\gamma$ is a Barbero-Immirzi-like parameter. From the variation of the action, the following equations of motion arise

$$
\begin{aligned}
\epsilon^{\alpha \mu \nu}\left(D_{\mu} e_{\nu}^{i}+\frac{1}{\gamma} F_{\mu \nu}^{i}\right) & =0, \\
\epsilon^{\alpha \mu \nu} F_{\mu \nu}^{i} & =0,
\end{aligned}
$$

where $D_{\mu} e_{\nu}^{i}=\partial_{\mu} e_{\nu}^{i}+\varepsilon^{i}{ }_{j k} A_{\mu}^{j} e_{\nu}^{k}$. These equations for different values of $\gamma$ represent a set of equations classically equivalent to three dimensional Einstein's theory, however, in spite of this equivalence we will see that the generalized $H J$ brackets depend on the $\gamma$ parameter, while in Palatini theory there is not such a dependence [38], in this sense the Palatini theory and PCS are different to each other. Along the paper, we will use the notation $\mu, \nu, \ldots=0,1,2$ for spacetime indices and the alphabet letters $a, b, c$ for space indices. Moreover, we will suppose that the manifold has topology $M=\Sigma \times R$, where $\Sigma$ is a Cauchy surface and $R$ is an evolution parameter. With these considerations at hand we perform the $2+1$ decomposition, and the action (1) takes the following form

$$
S[e, A]=\int \epsilon^{a b}\left[\left(e_{b}{ }^{i}+\frac{1}{\gamma} A_{b}{ }^{i}\right) \dot{A}_{a i}+\frac{1}{2} e_{0}^{i} F_{a b i}+A_{0 i}\left(D_{a} e_{b}{ }^{i}+\frac{1}{\gamma} F_{a b}{ }^{i}\right)\right] d^{3} x,
$$

we have removed an overall factor of 2 which does not affect to the equations of motion and we have defined $\epsilon^{0 a b} \equiv \epsilon^{a b}$. Moreover, here we have used

$$
\begin{aligned}
F_{a b i} & =\partial_{a} A_{b i}-\partial_{b} A_{a i}+\varepsilon_{i}{ }^{j k} A_{a j} A_{b k}, \\
D_{a} e_{b i} & =\partial_{a} e_{b i}+\varepsilon_{i}{ }^{j k} A_{a j} e_{b k} .
\end{aligned}
$$

The action (3) has been analyzed by using the Dirac and Faddeev-Jackiw approaches in 28, 37]; in these works the Dirac and Faddeev-Jackiw constraints, a symplectic tensor and the symmetries of the theory were reported. However, in the present paper we will extend those works by performing a $H J$ analysis and we will reproduce in more economical way those results. Furthermore, we identify the canonical momenta $\left(\Pi_{i}^{\mu}, p^{\mu}{ }_{i}\right)$ conjugated to $\left(A_{\mu}^{i}, e^{i}{ }_{\mu}\right)$

$$
\Pi_{i}^{\mu} \equiv \frac{\partial \mathcal{L}}{\partial \dot{A}_{\mu}^{i}}, \quad p^{\mu}{ }_{i} \equiv \frac{\partial \mathcal{L}}{\partial \dot{e}^{i}{ }_{\mu}},
$$


Hence, by using the momenta, from the action (3) we identify the following $H J$ Hamiltonians

$$
\begin{aligned}
H^{\prime} & \equiv \Pi+H_{0}=0, \\
\phi_{i} & \equiv p_{i}^{0}=0, \\
\tilde{\phi}_{i} & \equiv \Pi_{i}^{0}=0, \\
\varphi_{i}^{a} & \equiv p_{i}^{a}=0, \\
\tilde{\varphi}_{i}^{a} & \equiv \Pi_{i}^{a}-\epsilon^{a b}\left(e_{b i}+\frac{1}{\gamma} A_{b i}\right)=0,
\end{aligned}
$$

where $\Pi=\partial_{0} S$, identifying to $S$ with the action and $H_{0}$ with the canonical Hamiltonian

$$
H_{0}=-\frac{\epsilon^{a b}}{2} e_{0}^{i} F_{a b i}-A_{0}^{i}\left(D_{a} \Pi_{i}^{a}+\frac{1}{\gamma} \epsilon^{a b} \partial_{a} A_{b i}\right) .
$$

the definition of the momenta allows us to identify the fundamental Poisson brackets

$$
\begin{array}{r}
\left\{e_{\alpha}^{i}(x), p_{j}^{\mu}(y)\right\}=\delta_{\alpha}^{\mu} \delta_{j}^{i} \delta^{2}(x-y), \\
\left\{A_{\alpha}^{i}(x), \Pi_{j}^{\mu}(y)\right\}=\delta_{\alpha}^{\mu} \delta_{j}^{i} \delta^{2}(x-y) .
\end{array}
$$

In this manner, with the Hamiltonians identified, we construct the fundamental differential which describes the evolution of any function, say $f$, on the phase space [23 26]

$d f(x)=\int d^{2} y\left(\left\{f(x), H^{\prime}(y)\right\} d t+\left\{f(x), \varphi_{i}^{a}(y)\right\} d \xi_{a}^{i}+\left\{f(x), \tilde{\varphi}_{i}^{a}(y)\right\} d \tilde{\xi}_{a}^{i}+\left\{f(x), \phi_{i}(y)\right\} d \lambda^{i}+\left\{f(x), \tilde{\phi}_{i}\right\} d \tilde{\lambda}^{i}\right)$,

here, $\xi_{a}^{i}, \tilde{\xi}_{a}^{i}, \lambda^{i}$, and $\tilde{\lambda}^{i}$ are parameters associated with the Hamiltonians. On the other hand, we observe that the Hamiltonians $\phi_{i}$ and $\tilde{\phi}_{i}$ are involutives and $\varphi_{i}^{a}, \tilde{\varphi}_{i}^{a}$ are non-involutives. Involutive Hamiltonians, are those whose Poisson brackets with all Hamiltonians, including themself, vanish; otherwise, they are called non-involutives. The presence of non-involutive Hamiltonians introduce the generalized $H J$ brackets defined by 23 26]

$$
\{A, B\}^{*}=\{A, B\}-\left\{A, H_{\bar{a}}^{\prime}\right\}\left(C_{\bar{a} \bar{b}}\right)^{-1}\left\{H_{\bar{b}}^{\prime}, B\right\},
$$

where $\left(C_{\bar{a} \bar{b}}\right)$ is the matrix whose entries are given by the Poisson brackets between non-involutives Hamiltonians and $\left(C_{\bar{a} \bar{b}}\right)^{-1}$ its inverse; that matrix takes the form

$$
C_{\alpha \beta}=\left(\begin{array}{cc}
0 & \epsilon^{a b} \delta_{i j} \\
\epsilon^{b a} \delta_{i j} & -\frac{2}{\gamma} \epsilon^{a b} \delta_{i j}
\end{array}\right) \delta^{2}(x-y),
$$

with

$$
C_{\alpha \beta}^{-1}=\left(\begin{array}{cc}
-\frac{2}{\gamma} \epsilon_{a b} \delta^{i j} & \epsilon_{a b} \delta^{i j} \\
-\epsilon_{b a} \delta^{i j} & 0
\end{array}\right) \delta^{2}(x-y),
$$


hence, the generalized brackets between the fields read

$$
\begin{aligned}
\left\{e_{a}^{i}(x), e_{b}^{j}(y)\right\}^{*} & =-\frac{2}{\gamma} \epsilon_{a b} \delta^{i j} \delta^{2}(x-y), \\
\left\{e_{a}^{i}(x), A_{b}^{j}(y)\right\}^{*} & =\epsilon_{a b} \delta^{i j} \delta^{2}(x-y), \\
\left\{A_{a}^{i}(x), A_{b}^{j}(y)\right\}^{*} & =0 \\
\left\{e_{a}^{i}(x), \Pi_{j}^{b}(y)\right\}^{*} & =-\frac{1}{\gamma} \delta_{a}^{b} \delta_{j}^{i} \delta^{2}(x-y), \\
\left\{A_{a}^{i}(x), \Pi_{j}^{b}(y)\right\}^{*} & =\delta_{a}^{b} \delta_{j}^{i} \delta^{2}(x-y), \\
\left\{e_{a}^{i}(x), p_{j}^{b}(y)\right\}^{*} & =0 \\
\left\{\Pi_{a}^{i}(x), \Pi_{j}^{b}(y)\right\}^{*} & =0
\end{aligned}
$$

note that the fields $e^{\prime}$ s are noncommutative due to the presence of the $\gamma$ parameter. This fact makes PCS theory different to standard Palatini action where the triad is commutative [38]. With the generalized brackets at hand, we introduce the new fundamental $H J$ differential

$$
d f(x)=\int d^{2} y\left[\left\{f(x), H^{\prime}(y)\right\}^{*} d t+\left\{f(x), \phi_{i}(y)\right\}^{*} d \lambda^{i}+\left\{f(x), \tilde{\phi}_{i}(y)\right\}^{*} d \tilde{\lambda}^{i}\right],
$$

and we observe that the non-involutive Hamiltonians has been removed. On the other hand, the Frobenius integrability conditions on the Hamiltonians $\phi_{i}$ and $\tilde{\phi}_{i}$ introduce new $H J$ Hamiltonians. In fact, integrability conditions are relevant because ensure that the system (8) is integrable. From the integrability conditions the following Hamiltonians arise

$$
\begin{aligned}
\left.d \phi_{i}(x)=\int d^{2} y\left[\left\{\phi_{i}(x)\right), H^{\prime}(y)\right\} d t+\left\{\phi_{i}(x), \phi_{j}(y)\right\} d \lambda^{j}+\left\{\phi_{i}(x), \tilde{\phi}_{j}(y)\right\} d \tilde{\lambda}^{j}\right] & =0 \\
\rightarrow \tau_{i} \equiv \epsilon^{a b} F_{a b i} & =0 \\
d \tilde{\phi}_{i}(x)=\int d^{2} y\left[\left\{\tilde{\phi}_{i}(x), H^{\prime}(y)\right\} d t+\left\{\tilde{\phi}_{i}(x), \phi_{j}(y)\right\} d \lambda^{j}+\left\{\tilde{\phi}_{i}(x), \tilde{\phi}_{j}(y)\right\} d \tilde{\lambda}^{j}\right] & =0 \\
\rightarrow \tilde{\tau}_{i} \equiv D_{a} \Pi_{i}^{a}+\frac{1}{\gamma} \epsilon^{a b} \partial_{a} A_{b i} & =0 .
\end{aligned}
$$

The generalized algebra between the new Hamiltonians $\tau_{i}$ and $\tilde{\tau}_{i}$ is given by

$$
\begin{aligned}
& \left\{\tau_{i}, \tau_{j}\right\}^{*}=0, \\
& \left\{\tau_{i}, \tilde{\tau}_{j}\right\}^{*}=\varepsilon_{i j k} \tau^{k}, \\
& \left\{\tilde{\tau}_{i}, \tilde{\tau}_{j}\right\}^{*}=\varepsilon_{i j k} \tilde{\tau}^{k},
\end{aligned}
$$

where we can observe that these Hamiltonians are involutive, therefore we do not expect new Hamiltnonians. Furthermore, the Hamiltonians $\tau_{i}$ and $\tilde{\tau}_{i}$ form a Poincaré algebra. In fact, $\tau_{i}$ is related to translations and $\tilde{\tau}_{i}$ is related to rotations. With all involutive Hamiltonians at hand we construct the following generalized differential

$$
\begin{aligned}
d f(x) & =\int d^{2} y\left[\left\{f(x), H^{\prime}(y)\right\}^{*} d t+\left\{f(x), \phi_{i}\right\}^{*} d \lambda^{i}(y)+\left\{f(x), \tilde{\phi}_{i}(y)\right\}^{*} d \tilde{\lambda}^{i}+\left\{f(x), \tau_{i}(y)\right\}^{*} d \Upsilon^{i}(y)\right. \\
& \left.+\left\{f(x), \tilde{\tau}_{i}(y)\right\}^{*} d \tilde{\Upsilon}^{j}\right]
\end{aligned}
$$

where $\Upsilon^{i}$ and $\tilde{\Upsilon}^{i}$ are parameters related with the Hamiltonians $\tau_{i}$ and $\tilde{\tau}_{i}$ respectively. In this manner, from the fundamental differential we can calculate the characteristic equations [23 26], which will 
reveal the symmetries of the theory. The characteristic equations are given by

$$
\begin{aligned}
d e_{0}^{i} & =d \lambda^{i} \\
d A_{0}^{i} & =d \tilde{\lambda}^{i}, \\
d e_{a}^{i} & =\left(\partial_{a} e_{0}^{i}+\varepsilon_{l}^{i}{ }_{k} e_{0}^{l} A_{a}^{k}+\varepsilon_{l}^{i}{ }_{k} A_{0}^{l} e_{a}^{k}\right) d t-2 D_{a} d \Upsilon^{i}-\varepsilon_{j}{ }^{i l} e_{a l} d \tilde{\Upsilon}^{j}, \\
d A_{a}^{i} & =F_{a 0}^{i} d t-D_{a} d \tilde{\Upsilon}^{i} .
\end{aligned}
$$

hence, from the temporal part we identify the equations of motion

$$
\begin{aligned}
\partial_{0} e_{a}^{i} & =\partial_{a} e_{0}^{i}+\varepsilon_{l}^{i}{ }_{k} e_{0}^{l} A_{a}^{k}+\varepsilon_{l}{ }_{k} A_{0}^{l} e_{a}^{k}, \\
\partial_{0} A_{a}^{i} & =\partial_{a} A_{0}^{i}-\varepsilon_{l j}{ }^{i} A_{0}^{l} A_{a}^{j},
\end{aligned}
$$

which correspond to 3d Einstein's equations. On the other hand, we observe from the characteristic equations that the fields $e_{0}^{i}$ and $A_{0}^{i}$ are not related neither $t$ nor $\Upsilon^{i}$ and $\tilde{\Upsilon}^{i}$ parameters, which means that they are identified as Lagrange multipliers. Moreover, the parameters associated with the involutives Hamiltonians are related to the following gauge transformations

$$
\begin{array}{r}
\delta e_{a}^{i}=D_{a} \delta \Upsilon^{i}+\frac{1}{2} \varepsilon_{j}{ }^{i l} e_{a l} \delta \tilde{\Upsilon}^{j} \\
\delta A_{a}^{i}=\frac{1}{2} D_{a} \delta \tilde{\Upsilon}^{i} .
\end{array}
$$

We can observe that similar results were reported in [37], where different approaches were used, however, we can note that the $H J$ approach is an economical way for finding the symmetries of the theory.

We finish this section by performing the counting of physical degrees of freedom: in this formalism the physical degrees of freedom are identified with the dynamical fields found in the characteristic equations minus the complete set of involutive Hamiltonians. For this theory, the dynamical variables are the following six $e_{a}^{i}$ and six $A_{a}^{i}$; the involutive Hamiltonians are $12\left(\phi_{i}, \tilde{\phi}_{i}, \tau_{i}, \tilde{\tau}_{i}\right)$, and thus $D F=12-12=0$, the theory is devoid of physical degrees of freedom, as expected.

In the following section, we will complete our analysis by performing the canonical covariant method of the PCS theory.

\section{CANONICAL COVARIANT ANALYSIS}

We start our study by taking the variation of the action (11) with respect the dynamical fields,

$$
\delta S[e, A]=\int_{M}\left[\left(\varepsilon^{\alpha \mu \nu}\left(D_{\mu} e_{\nu}^{i}+\frac{1}{\gamma} F_{\mu \nu}^{i}\right)\right) \delta A_{\alpha i}+\left(\varepsilon^{\alpha \mu \nu} F_{\mu \nu i}\right) \delta e_{\alpha}^{i}+\partial_{\mu}\left(\varepsilon^{\mu \alpha \nu}\left(e_{\alpha}^{i}+\frac{1}{\gamma} A_{\alpha}^{i}\right) \delta A_{\nu i}\right)\right] d x^{3}(15)
$$

where we identify the equations of motion (2) and from the divergence term a symplectic potential is identified [22]

$$
\Psi^{\mu}=\varepsilon^{\mu \alpha \nu}\left(e_{\alpha}^{i}+\frac{1}{\gamma} A_{\alpha}^{i}\right) \delta A_{\nu i} .
$$


In this manner, we define the essential object of the canonical covariant method, the covariant phase space; the covariant phase space for the theory described by (1) is the space of solutions of the equations of motion (2), and we will call it $Z$ [22, 30]. Hence, on $Z$ the fields $A_{\mu}^{i}$ and $e_{\mu}^{i}$ are zeroforms and its variations (exterior derivation on $Z$ ) $\delta A_{\mu}^{i}$ and $\delta e_{\mu}^{i}$ are 1-forms. Therefore, the variation of the symplectic potential generates the two form symplectic structure,

$$
\omega=\int_{\Sigma} J^{\mu} d \Sigma_{\mu}=\int_{\Sigma} \delta \Psi^{\mu} d \Sigma_{\mu}=\int_{\Sigma} \varepsilon^{\mu \alpha \nu} \delta\left(e_{\alpha}^{i}+\frac{1}{\gamma} A_{\alpha}^{i}\right) \wedge \delta A_{\nu i} d \Sigma_{\mu}
$$

where $\Sigma$ is a Cauchy surface. We will find the symmetries of the theory trough that geometric structure. In fact, we will prove that $\omega$ is closed and gauge invariant; the closeness of $\omega$ is equivalent to the Jacobi identity that Poisson brackets satisfy in the Hamiltonian scheme. In addition, we know that gauge invariance is reflection of an internal symmetry when the theory is singular. Furthermore, the integral kernel $J^{\mu}$ of the geometric form, is conserved; this fact will be important because it guarantees that $\omega$ is independient of $\Sigma$. Hence, we observe that $\delta^{2} e^{i}{ }_{\mu}=0$ and $\delta^{2} A_{\alpha}^{i}=0$, due to $e^{i}{ }_{\mu}$ and $A_{\alpha}^{i}$ are independent zero forms on $Z$ and $\delta$ is nilpotent, therefore $\omega$ is closed. Now, we shall find the linearized equations of motion; they are obtained from the substitutions $A_{\mu}^{i} \rightarrow A_{\mu}^{i}+\delta A_{\mu}^{i}$, and $e_{\mu}^{i} \rightarrow e_{\mu}^{i}+\delta e_{\mu}^{i}$ into the equations of motion, and keeping only the first order terms, hence

$$
\begin{aligned}
\epsilon^{\alpha \mu \nu} D_{\mu} \delta A_{\nu i} & =0, \\
\epsilon^{\alpha \mu \nu}\left(D_{\mu} \delta A_{\nu}^{i}+\varepsilon_{i j k} \delta A_{\mu}^{j} e_{\nu}^{k}\right) & =0,
\end{aligned}
$$

the linearized equations will be important for proving the conservation of $J^{\mu}$. In fact, by taking the generator of rotations in the gauge transformations (14) and under an arbitrary variation we obtain

$$
\begin{gathered}
\delta e_{\mu}{ }_{\mu}^{i}=\delta e_{\mu}^{i}+\frac{1}{2} \varepsilon_{j}{ }^{i l} \delta e_{\mu l} \epsilon^{j}, \\
\delta A_{\mu}^{\prime i}=\delta A_{\mu}^{i}+\frac{1}{2} \varepsilon^{i}{ }_{j k} \delta A_{\mu}^{j} \epsilon^{k},
\end{gathered}
$$

where we have called $\epsilon^{i} \equiv d \Upsilon^{i}$ and $\tilde{\epsilon}^{i} \equiv d \tilde{\Upsilon}^{i}$. In this manner, under the transformations (19) the symplectic structure transforms as

$$
\begin{aligned}
\omega^{\prime} & =\int_{\Sigma}\left(\varepsilon^{\mu \alpha \nu} \delta e_{\alpha}^{\prime i} \wedge \delta A_{\nu i}^{\prime}+\frac{1}{\gamma} \varepsilon^{\mu \alpha \nu} \delta A_{\alpha}^{\prime i} \wedge \delta A_{\nu i}^{\prime}\right) d \Sigma_{\mu}, \\
& =\omega+\int_{\Sigma} O\left(\epsilon^{2}\right),
\end{aligned}
$$

thus, $\omega$ is a $S U(2)$ singlet. Hence, this fact allows us prove the conservation of $J^{\mu}$, this is

$$
\begin{aligned}
\partial_{\mu} J^{\mu} & =D_{\mu} J^{\mu} \\
& =\epsilon^{\mu \alpha \nu} D_{\mu} \delta e_{\alpha}^{i} \wedge \delta A_{\nu i}+\epsilon^{\mu \alpha \nu} \delta e_{\alpha}^{i} \wedge D_{\mu} \delta A_{\nu i}+\frac{1}{\gamma} \epsilon^{\mu \alpha \nu} D_{\mu} \delta A_{\alpha}^{i} \wedge \delta A_{\nu i}+\frac{1}{\gamma} \epsilon^{\mu \alpha \nu} \delta A_{\alpha}^{i} \wedge D_{\mu} \delta a_{\nu i}, \\
& =0
\end{aligned}
$$

where we have used the linearized equations of motion (18) and the antisymmetry of the 1-forms $\delta e_{\mu}^{i}$ and $\delta A_{a}^{i}$.

On the other hand, we know that both Palatini and Chern-Simons theories are diffeomorphism 
covariant, and this important symmetry must to be contained in the fundamental gauge transformations. In fact, with the particular choice

$$
\delta \Upsilon^{i}=\epsilon^{\rho} e_{\rho}^{i} \quad \text { and } \quad \delta \tilde{\Upsilon}^{i}=2 \epsilon^{\mu} A_{\mu}^{i},
$$

from the gauge transformations (14) we obtain

$$
\begin{gathered}
\delta e_{\alpha}^{\prime i}=\delta e_{\alpha}^{i}+\mathcal{L}_{\vec{\epsilon}} \delta e_{\alpha}^{i}, \\
\delta A_{\alpha}^{\prime i}=\delta A_{\alpha}^{i}+\mathcal{L}_{\vec{\epsilon}} \delta A_{\alpha}^{i}
\end{gathered}
$$

this means that diffeomorphisms are identified as internal symmetry of the theory. In this manner, we can prove that the symplectic structure transforms under (22) as

$$
\begin{aligned}
\omega^{\prime} & =\int_{\Sigma} \varepsilon^{\alpha \beta \mu}\left(\delta e_{\beta}^{\prime i}+\frac{1}{\gamma} \delta A_{\beta}^{\prime i}\right) \wedge \delta A_{\mu i}^{\prime} d \Sigma_{\alpha}, \\
& =\omega+\int_{\Sigma} \mathcal{L}_{\bar{\epsilon}} \omega
\end{aligned}
$$

however, $\mathcal{L}_{\vec{\epsilon}} \omega=\vec{\epsilon} \cdot d \omega+d(\vec{\epsilon} \cdot \omega)$, but $\delta \omega=0$ (it is closed) and, the term $d(\vec{\epsilon} \cdot \omega)$ corresponds to a surface term. Therefore $\omega$ is invariant under infinitesimal diffeomorphisms.

Once we have found the symmetries of the theory from the symplectic point of view, we consider that upon picking $\Sigma$ to be the standard initial value surface $t=0$, the symplectic structure takes the standard form

$$
\omega=\int_{\Sigma} \delta \Pi_{i}^{a} \wedge \delta A_{a}^{i}
$$

where $\Pi_{i}^{a}=\varepsilon^{b a}\left(e_{i b}+\frac{1}{\gamma} A_{b i}\right)$. In this manner, under these considerations, we are able to perform a Hamiltonian study. In fact, let us consider to $f$ as any 0 -form defined on $Z$, hence the Hamiltonian vector field defined by the symplectic form (24) is given by

$$
X_{f} \equiv \int_{\Sigma} \frac{\delta f}{\delta \Pi_{i}^{a}} \frac{\delta}{A_{a}^{i}}-\frac{\delta f}{\delta A_{a}^{i}} \frac{\delta}{\delta \Pi_{i}^{a}} .
$$

Moreover, the Poisson bracket between two zero-forms is defined as usual

$$
\{f, g\}_{P} \equiv-X_{f} g=\int_{\Sigma} \frac{\delta f}{A_{a}^{i}} \frac{\delta g}{\delta \Pi_{i}^{a}}-\frac{\delta f}{\delta \Pi_{i}^{a}} \frac{\delta g}{\delta A_{a}^{i}} .
$$

Then, if we smearing the constraints with test fields, namely

$$
\begin{array}{r}
\tau\left[N^{i}\right]=\int_{\Sigma} N^{i}\left[\varepsilon^{a b} F_{a b i}\right], \\
\tilde{\tau}\left[M^{i}\right]=\int_{\Sigma} M^{i}\left[D_{a} \Pi_{i}^{a}+\frac{1}{\gamma} \varepsilon^{a b} \partial_{a} A_{b i}\right],
\end{array}
$$

and we calculate the fundamental variations of these constraints

$$
\frac{\delta \tau}{\delta A_{a}^{i}}=2 \varepsilon^{a b} \partial_{b} N_{i}+2 \varepsilon_{i l}^{k} \varepsilon^{a b} A_{b}^{l} N_{k}, \quad \frac{\delta \tau}{\delta \Pi_{i}^{a}}=0,
$$

and

$$
\frac{\delta \tilde{\tau}}{\delta A_{a}^{i}}=M^{k} \varepsilon_{i k}^{j} \Pi_{i}^{a}+\frac{1}{\gamma} \varepsilon^{a b} \partial_{b} M^{i}, \quad \frac{\delta \tilde{\tau}}{\delta \Pi_{i}^{a}}=-D_{a} M^{i}
$$


this allows us to calculate the following Poisson brackets between the constraints and the fields

$$
\begin{aligned}
& \left\{A_{a}^{i}, \tau\right\}=0 \\
& \left\{A_{a}^{i}, \tilde{\tau}\right\}=-D_{a} M^{i}, \\
& \left\{\Pi_{i}^{a}, \tau\right\}=-\varepsilon^{a b} \partial_{b} N_{i}-\varepsilon^{k}{ }_{i l} \varepsilon^{a b} A_{b}^{l} N_{k}, \\
& \left\{\Pi_{i}^{a}, \tilde{\tau}\right\}=-M^{k} \varepsilon_{k i}{ }^{j} \Pi_{j}^{a}-\frac{1}{\gamma} \varepsilon^{a b} \partial_{b} M_{i} .
\end{aligned}
$$

In this manner, by taking into account the equations (28), we observe that the motion generated by $\tau[N]$ and $\tilde{\tau}_{i}[M]$ is

$$
\begin{aligned}
A_{a}^{\prime} i & \longrightarrow A_{a}^{i}+D_{a} M^{i} \epsilon \\
\Pi_{i}^{\prime}{ }^{a} & \longrightarrow \Pi_{i}^{a}+M^{k} \varepsilon_{k i}{ }^{j} \Pi_{j}^{a} \epsilon+\frac{\epsilon}{\gamma} \varepsilon^{a b} \partial_{b} M^{i}+\epsilon \epsilon^{a b} D_{b} N_{i},
\end{aligned}
$$

where $\epsilon$ corresponds to an infinitesimal parameter [22, 30]. The transformations of the connection are those found by means the $H J$ approach. However, it is important to comment some differences between the approaches used in this paper. We can note that in the $H J$ method the dynamical variables are given by the connection and the triad fields, in this sense, the $H J$ method is similar to perform a pure Dirac's method [38] in which the canonical momenta are associated with all dynamical variables. On the other hand, in the canonical covariant method the dynamical variables are those occurring in the action with time derivative (see the action (3) ) and only those variables are associated with its canonical momenta. In this respect, in the canonical covariant method we will not find any gauge transformations associated with the triad field, since from the beginning it is not a dynamical variable. In addition, in order to calculate the gauge transformations we used the constraints found in the $H J$ approach. In fact, we know that in the canonical covariant method we have not control on the constraints and this fact restricts us to perform the counting of physical degrees of freedom and the construction of a generalized bracket such as in the $H J$ scheme is done.

\section{CONCLUSSIONS}

A detailed $H J$ and canonical covariant analysis for $P C S$ theory were developed. With respect to the $H J$ study, we have constructed a generalized differential given in terms of the generalized brackets and involutive Hamiltonians allowing us identify the characteristic equations of the theory. The contribution of the $\gamma$ parameter is observed in the generalized brackets: the triad becomes to be noncommutative and this fact makes PCS different at classical level from Palatini theory. Moreover, the gauge transformations were reported and the counting of physical degrees of freedom was performed. On the other hand, from the symplectic point of view, a closed and gauge invariant geometric structure was constructed and the symmetries of the theory were identified. We would point out that in this formalism we have not control on the constraints of the theory, and this fact do not allow us to construct a kind of generalized brackets such as in the $H J$ scheme is done; in this respect the contribution of $\gamma$ is missed. In this manner, the $H J$ framework show advantages with 
respect to the canonical covariant formalism. Therefore, we have extended the results presented in [28, 37] where different approaches were used.

\section{Acknowledgements}

We would like to thank R. Cartas-Fuentevilla for discussion on the subject and reading of the manuscript.

[1] D.M. Gitman, I.V. Tyutin, Quantization of Fields with Constraints, in: Springer Series in Nuclear and Particle Physics, Springer, (1990).

[2] M.B. Green, J.H. Schwarz, E. Witten, Super String Theory (Cambridge University Press, Cambridge, 1986).

[3] J. Polchinski, String Theory (Cambridge University Press, Cambridge, 1998).

[4] C. Rovelli, Quantum Gravity (Cambridge University Press, Cambridge, 2004).

[5] T. Thiemann, Modern Canonical Quantum General Relativity (Cambridge University Press, Cambridge, 2007).

[6] A. Hanson, T. Regge, C. Teitelboim, Constrained Hamiltonian Systems, Accademia Nazionale dei Lincei, Roma, (1978).

[7] L.D. Faddeev, R. Jackiw, Phys. Rev. Lett. 60, 1692, (1988) .

[8] E. M. C. Abreu, A.C.R. Mendes, C. Neves, W. Oliveira, F.I. Takakura, L.M.V. Xavier, Modern Phys. Lett. A 23, 829, (2008).

[9] E.M.C. Abreu, A.C.R. Mendes, C. Neves, W. Oliveira, F.I. Takakura, Inter. J. Modern Phys. A 22, 3605, (2007).

[10] E.M.C. Abreu, C. Neves, W. Oliveira, Internat. J. Modern Phys. A 21, 5329, (2008).

[11] C. Neves, W. Oliveira, D.C. Rodrigues, C. Wotzasek, Phys. Rev. D 69, 045016, (2004).

[12] C. Neves, C. Wotzasek, Internat. J. Modern Phys. A 17, 4025, (2002).

[13] C. Neves, W. Oliveira, Phys. Lett. A 32, 267, (2004).

[14] J.A. Garcia, J.M. Pons, Internat. J. Modern Phys. A 12, 451, (1997) .

[15] E.M.C. Abreu, A.C.R. Mendes, C. Neves, W. Oliveira, R.C.N. Silva, C. Wotzasek, Phys. Lett. A 37, 3603-3607, (2010).

[16] L. Liao, Y.C. Huang, Ann. Phys. 322, 2469-2484, (2007).

[17] A. Escalante, J. Manuel-Cabrera, Ann. Physics. 343, 27-39, (2014).

[18] A. Escalante, M. Zárate, Annals. Phys. 353, 163-178, (2015)

[19] A. Escalante, J. Manuel-Cabrera, Annals. Phys. 36, 1585-604, (2015).

[20] A. Escalante, O. Rodríguez-Tzompantzi, Annals. Phys. 364, 136 (2016).

[21] J. Barcelos-Neto, C. Wotzasek, Modern Phys. Lett. A 7 (19) (1992) 1737-1747; J. Barcelos-Neto, C. Wotzasek, Internat. J. Modern Phys. A 7 (20) (1992) 4981-5003.

[22] C. Crnkovi'c and E. Witten, in Three Hundred Years of Gravitation, eds. S. W. Hawking and W. Israel (Cambridge University Press, Cambridge, (1987)).

[23] Y. Güler, On the dynamics of singular continuous systems, J. Math. Phys. 30785 (1989).

[24] C. Caratheodory, Calculus of Variations and Partial Diferential equations of the First Order, 3rd edn (American Mathematical Society) (1999). 
[25] M.C. Bertin, B.M. Pimentel, C.E. Valcárcel, Ann. Phys. 3233137 (2008).

[26] M.C. Bertin, B.M. Pimentel, C.E. Valcárcel, J. Math. Phys. 55112901 (2014).

[27] N. T. Maia, B.M. Pimentel, C.E. Valcárcel, Class. Quantum Grav. 32, 185013, (2015).

[28] V. Bonzom and E. R. Livine, Class. Quant. Grav, vol. 25, no. 19, 195024, (2008).

[29] M. Montesinos, Class. Quant. Grav. 23: 2267-2278, (2006).

[30] A. Escalante, Phys. Lett. B 676, 105-111, (2009) .

[31] A. Escalante and C. Medel-Portugal, Annals of Phys. 391, 27-46, (2018).

[32] S. Holts, Phys. Rev. D 53, 5966 (1996).

[33] M. Montesinos, Class.Quant.Grav. 18, 1847-1852, (2001).

[34] D. Jimenez-Rezende, A. Perez, Phys. Rev. D79: 064026, (2009).

[35] A. Escalante, J. Manuel-Cabrera, Ann. Phys. 361, 585?604 (2015).

[36] A. Escalante, J. Manuel-Cabrera. Eur. Phys. J. C 77, 303 (2017)

[37] A. Escalante, Prihel-Cavildo-Sánchez, Adv.Math.Phys. 3474760, (2018).

[38] A. Escalante, O. Rodríguez-Tzompantzi, J. High Energy Phys. 05, 073, (2014). 